\title{
A computer-automated laboratory for studying complex perception-action skills
}

\author{
RALPH J. ROBERTS, JR., DUNCAN BROWN, SCOTT WIEBKE, and MARSHALL M. HAITH \\ University of Denver, Denver, Colorado
}

\begin{abstract}
In this paper, we describe a computer-based laboratory for collecting and analyzing real-time performance and eye-movement data by using video game-like tasks. These tasks assess skills that require the integration of varying numbers of perception and action components. Customized hardware and software are described for configuring tasks, collecting data, reconstructing performance, and organizing, editing, and reducing data. General design considerations are emphasized for solving several of the problems encountered in constructing a real-time data collection system. The paper concludes with examples of data that illustrate the system's usefulness for studying complex perception-action skills.
\end{abstract}

In this paper, we describe a computer-based laboratory for collecting and analyzing real-time performance data as well as synchronized eye-tracking data using video game-like tasks. Customized hardware and software are described for designing experimental tasks, collecting data, reconstructing performance, and organizing, editing, and reducing data. The general capacities of the system are presented, as are the strategies developed for solving several of the problems that the researcher must face when building a real-time data-collection/analysis system. Although the specifics of the system should be of particular interest to researchers interested in perceptionaction skills, the general approach to data collection, organization, and analysis should be informative for a variety of researchers interested more generally in real-time data collection. ${ }^{1}$

The paper begins with a brief review of some existing technologies used to study perception-action skills, in which we show how the present system builds on previous approaches but differs from them in several important ways. Three sections then follow. An overview of the laboratory is presented first, along with a description of the types of tasks employed, the general capabilities of the system, and a hypothetical experimental session. In the next two sections, the hardware and software are described in greater detail. We emphasize the strategies used to make the system as powerful and flexible as possible, given the limits of money and time. We conclude by presenting sample data to illustrate the system's usefulness for studying complex perception-action skills.

Support for this work was provided by NSF Grant BNS 86108043 to R. J. Roberts, Jr., the MacArthur Foundation's Early Childhood Transitions Network, a University of Denver Biomedical Research grant to R. J. Roberts, Jr., and an NIMH Research Scientist Award to M. M. Haith. The authors thank Naomi Wentworth and Rick Canfield for their help with the eye-movement component of the system. Reprints can be obtained by writing Ralph J. Roberts, Jr., Department of Psychology, University of Denver, Denver, CO 80208 (E-mail address: RROBERTS@DUCAIR).

\section{BACKGROUND}

For at least a century, the attempt to understand how people learn and carry out complex perception-action skills has presented a daunting challenge for scientists interested in human behavior. Such skills are thought to involve an on-line strategic integration of several components of action and perception (see, e.g., Arbib, 1980; Hofsten, 1985; Turvey \& Carello, 1988). Examples include the operation of a vehicle, performance on a musical instrument, and the playing of a sport. One problem in studying such skills is the difficulty of collecting data that capture the natural complexity of performance but that are also amenable to later analysis.

Early studies of the real-time dynamics of skilled action were conducted by Bryan and Harter (1899) on telegraphers and by Book (1908) on typists. These researchers used paper-and-ink event recorders to document the sequence and timing of keypresses. Although they were clearly ahead of their time in developing methods for real-time data collection, conceptual and technological limitations made it difficult for them to take advantage of the data. Unfortunately, subsequent research on skilled action was focused almost exclusively on greatly simplified tasks, such as pointing or pursuit-rotor tasks. These tasks were often chosen because performance could be measured easily and because the principles governing performance could be understood in terms of the behavioristic theories of the day.

With the "cognitive revolution" of the 1960 s and 1970s, interest in more complex skills increased. In fact, in some of the earliest arguments against the adequacy of behavioristic learning principles, typing and piano playing were used as examples of behavior whose sequential properties were difficult to explain without one's positing "unseen" cognitive entities (Lashley, 1951). During the past 2 decades, researchers have examined the realtime dynamics of several complex perception-action skills, including typing (e.g., Cooper, 1983), computer 
use (e.g., Card, Moran, \& Newell, 1983), and piano playing (e.g., Schaffer, 1981). These researchers have used computers, photography, and video recording, as well as combinations of these technologies, to capture performance.

The availability of microcomputers provides new opportunities for studying skill. Computers can digitize, store, and process large amounts of data relatively quickly and inexpensively, and thus they have been used for examining skills such as typing and piano playing. Computer-video hybrid systems have been developed to record and analyze limb movements (e.g., trajectories and acceleration) in two- and three-dimensional space (e.g., the WATSMART system). These systems are used by sport scientists interested in the biomechanics of skilled performance (Winter, 1979) and by psychologists interested in reaching, catching, posture control, and locomotion (e.g., Benson, 1990; Hofsten \& Ronnqvist, 1988).

The system described here is also a computer-video system designed for studying complex perception-action skill, but it differs from other existing systems in several respects. First, most current systems are best suited for skills whose specific sequence of actions is fairly well prescribed, such as typing from a text, reading music from a score, or performing a specific gymnastic move. In the present case, we sought to develop a task context in which the sequencing and timing of action would be more improvisational, requiring the person to adapt to changing and somewhat unpredictable events, such as the driving of a car or the playing of tennis. Second, most current systems for studying perception-action skills have been designed for the study of either perception or action exclusively, and they rarely permit one to examine both simultaneously. The present system was designed so that the perceptual input could be varied and somewhat unpredictable while both perceptual processing (via eye movements) and skilled action were being evaluated. Our goal here is not to suggest that researchers should duplicate the specific system we have built. Rather, we hope to provide an example of one approach to building such a system and a general guide to the issues involved in developing a research platform for studying complex perceptionaction skills.

\section{OVERVIEW OF THE LABORATORY SETUP}

\section{Tasks}

We wanted to develop tasks in which skilled action could be complex and somewhat improvisational so that expert-level performance would take considerable time to develop and would involve the orchestration or integration of several components of action and perception. Successful action should not be scripted, but should require on-line decisions concerning the sequence and timing of actions. Such decisions should be based on the perceptual information available from a changing task environment. In addition, the task should be amenable to collecting data on eye movements, on the relevant actions of the performer, and on the changing features of the task.
The tasks that we developed to fit these criteria are based on the video game concept. Specific types of video games provide a potentially ideal context for studying perception-action skills (cf. Donchin, 1989). Expertise at video games can often only be acquired with considerable practice, and it requires the orchestration of several components of action and perception. Video games present simulated dynamic environments in which players control the actions of one or more objects. While a high degree of precision in the timing and sequencing of action is usually necessary, actions cannot be scripted, since the task environment is not entirely predictable.

\section{System Features}

The laboratory contains several features that we thought were critical for making the video game a viable research context. We describe the most important features here; their implementation will be described in later sections. The first feature is action realism. It is important that the video display have high spatial resolution and that the processing responsible for updating the display be relatively fast. Both are important for giving the player a sense that his or her actions have immediate consequences, as well as for providing the player with a degree of control that is commensurate with the player's actions. Displays with spatial resolutions that are too coarse or too slow produce a feeling of disconnectedness between the player's actions and the simulated objects controlled by those actions.

A second feature is task malleability. Commercially available video games are obviously not designed for experimental purposes, and without modification, they have limited usefulness as research tasks. The primary problem concerns the lack of experimental control over parameters of the game's functioning. A researcher must be able to change various aspects of the task, although it is cumbersome to rewrite program code every time a change is desired. Thus, we built the system so that the experimenter could design a variety of experimental tasks relatively easily without being required to do any new programming.

The third system feature is data resolution. Whenever a researcher collects data from an on-going process, decisions must be made about how frequently to sample data and what information to sample. Increases in sampling rate and informational detail usually afford better precision and veridicality but at the cost of increasing data storage and postcollection data processing. Making intelligent decisions about grain level requires consideration of the purpose of the research, the available technological tools, and the relative costs and benefits of alternative possibilities. For studying the real-time dynamics of skilled action, fine-grained detail in both time and performance is desirable for a precise representation of the timing and sequencing of action.

Closely related to degree of data resolution is the fourth feature, performance replay. There are innumerable variables one can examine that describe different aspects of complex, real-time, skilled activity. Although the researcher often knows a priori many of the variables that 
are most relevant to specific questions, exploratory analyses are often required to find the variable or pattern of variables that best represents aspects of the skill of interest. The ability to "reconstruct" performance as it occurred, or in slow motion, can significantly aid the researcher's efforts at understanding performance, especially with respect to skills that have not been studied extensively in the past. We designed the system with this capability.

\section{A Hypothetical Experimental Session}

The following scenario should help convey a general picture of our laboratory system and some of its capabilities.

After a study is designed, an experimenter runs several computer programs that configure the experimental tasks. These programs present menus that permit the specification of numerous task parameters and testing procedures, such as trial duration and the content of between-trial feedback. This information is stored in several configuration files.

Before testing, the experimenter adjusts a headrest for steadying the subject's head for recording eye position (see Figure 1). During testing, the subject and experimenter sit at different consoles. Task presentation and data collection are controlled from the experimenter's con- sole. The first task calibrates the eye-tracking system by presenting a matrix of dots at known locations on an otherwise blank screen. Using a corneal reflection system (see below), a computer collects 30 samples (at $60 \mathrm{~Hz}$ ) of the left eye's position while the subject fixates each dot.

Before a subject performs a given task, the experimenter runs two programs. The first program sets up the task on the subject's console, using the appropriate configuration information. A second program controls data collection. This program prompts the experimenter for information on the subject and session (e.g., subject number, date, condition, age). The program then starts data collection when the subject begins the task and temporarily halts collection during between-trial intervals. Three types of data are collected at a rate of $60 \mathrm{~Hz}$ : (1) the positions and identity of all objects on the task screen; (2) the status of the player's control buttons; and (3) eyeposition data that specify the direction of gaze of the subject's left eye. These data are stored in separate files with identifying file-name extensions that specify the type of data collected.

The subject performs three tasks, which are designed to assess how performers at different levels of expertise strategically orchestrate several components of action and perception. These skills include the selection of targets in a cluttered visual array, the judgment of intercept times

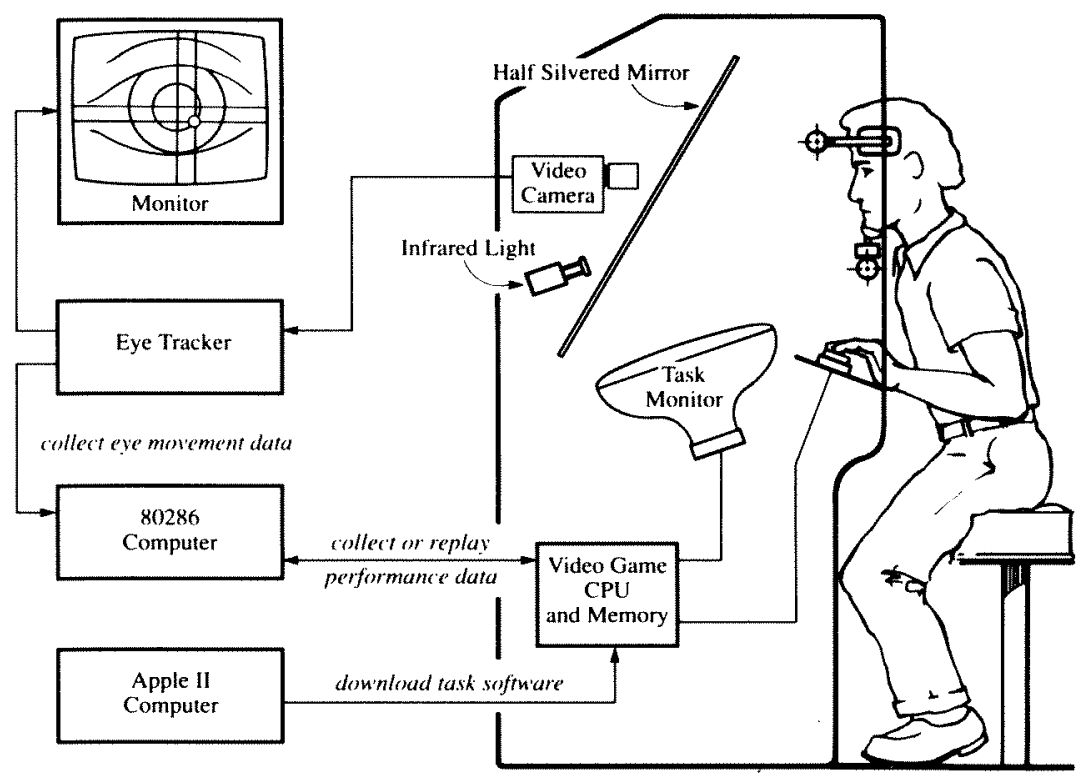

Figure 1. A schematic diagram of the data-collection/replay system. The cutaway of the video-game booth shows a subject viewing a reflection of the task monitor on a halfsilvered screen. Task software is downloaded from the Apple II + computer to the videogame RAM. A head- and chinrest steadies the subject's head. Behind the one-way mirror, an infrared-sensitive video camera focuses on the subject's left eye. A near-infrared light, also behind the mirror, illuminates the eye. The video signal from the camera is fed to an automatic eye tracker that outputs digital $x, y$ locations of the centers of the pupil and corneal reflection of the infrared light to an 80286 PC. The eye tracker also outputs an image of the eye to a video monitor with superimposed cross-hairs showing the calculated position of the centers of the pupil and corneal reflection. The $80286 \mathrm{PC}$ is connected to the video-game RAM and can either collect performance data during testing or, later, feed collected data back through the video game to replay performance. 
and positions between moving objects, and the control of the actions of a simulated vehicle. The three tasks vary in the number and type of component skills assessed.

The Fullgame task consists of 1030 -sec trials. The subject controls a triangular "ship" by pressing two buttons that rotate the ship around its center axis (clockwise or counterclockwise), a "thrust" button that moves the ship in the direction in which it is pointing, and a "fire"' button that releases shots that move across the screen (see Figure 2). Also displayed on the screen are 3-16 obstacles ("asteroids") that move across the screen in different straight-line trajectories and at different velocities. The objects "wrap around" to the opposite side when they reach an edge. The subject's task is to maneuver the ship so that the asteroids do not collide with the ship and to shoot as many asteroids as possible. The subject performs 10 trials, each lasting $30 \mathrm{sec}$. No two trials are identical, since the starting trajectories and velocities are determined randomly (within a range of values).

This task requires that the subject coordinate several components of perception and action, such as turning and thrusting movements, target selection, and intercept timing.

Next, the subject performs 128 trials of the Interception Component task. This task assesses a component skill used in the previous task (target interception) in a more constrained experimental setting. The ship faces straight up and cannot be rotated or moved (see Figure 2). During a trial, a single target comes from either the right or the left side of the top half of the screen and traverses the length of the screen at a constant speed. The subject's task is to time the release of one shot (via a buttonpress) to intercept the target. Across trials, the targets vary in size, speed, and distance from the ship. Performance and eye-movement data are collected as in the first task.

Finally, the Intercept Detection task assesses the subject's ability to use peripheral vision to select targets in visual scenes with varying amounts of "clutter." For each of 64 trials, the subject first fixates a bright spot at the center of an otherwise blank screen. After an auditory warning, several objects (similar to the asteroids) move across the screen from different positions, at different speeds and on different trajectories. The subject's task is to find the object, as quickly as possible, that would most likely intercept the center spot. Finding the object means looking directly at it. The trials vary in the number of objects displayed and in the trajectories and velocities of the objects. For this task, the computer only collects eyemovement and object-position data.

\section{SYSTEM HARDWARE}

\section{System Components}

We constructed the laboratory with the following components: an 8-MHz $80286 \mathrm{PC}$, an Apple II + microcomputer, an ISCAN corneal reflection eye tracker, a modified arcade-style video game (Asteroids, by Atari), and several custom-built circuit boards and cables. The 80286 PC contains 4.5 MB of RAM, an EGA graphics monitor, and a $40-\mathrm{MB}$ hard drive. The Apple $I+$ has $64 \mathrm{~K}$ of RAM and two floppy-disk drives. The video game uses a 6502 processor, a graphics coprocessor, system ROM and RAM totaling $12 \mathrm{~K}$, a 19 -in. $x, y$ vector monochrome monitor with 1,024 (horizontal) $\times 768$ pixel (vertical) resolution, and four buttons for player input.

We used a commercial arcade-style video game for several reasons. First, these games have been designed for high-speed interactive graphics processing. The Asteroids hardware uses a fast graphics coprocessor to draw on an $x, y$ vector graphics monitor, freeing the main game processor from this task. The combination of an $x, y$ monitor and a special-purpose graphics coprocessor results in very fast high-resolution graphics processing. In addition, the main game processor is optimized for
Fullgame Task

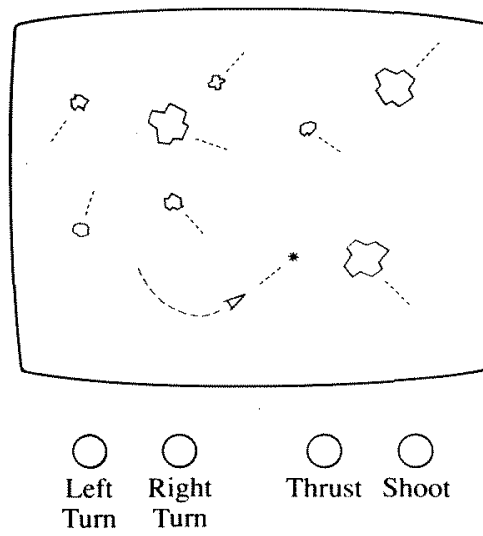

Interception Component Task

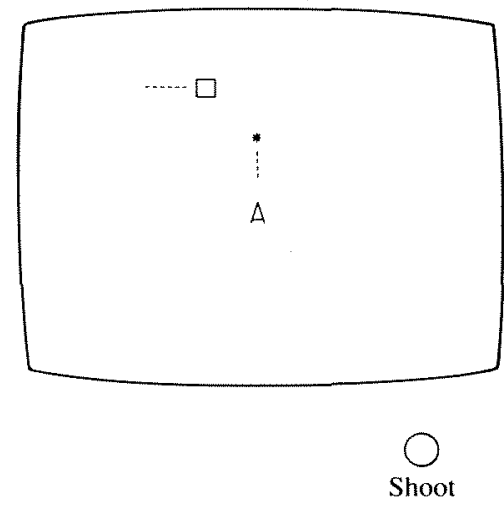

Figure 2. A representation of the screen displays for the Fullgame task and the Component Interception task. The dotted lines are not displayed on the screen but are shown here to represent the motions of the objects. The circles at the bottom of the figure show the relative positions of the buttons that the subject uses for each task. 
assembly-language video-game software, without the overhead of a general operating system (such as MS-DOS).

Another reason for using this equipment was cost. Since arcade-style video games were very popular several years ago but are currently much less so, used hardware is abundant and inexpensive. For example, a used Asteroids game sells for $\$ 50-\$ 150$ (a new 19-in. $x, y$ vector monitor alone can cost several thousand dollars). We also felt comfortable using this equipment, since one of us had considerable expertise in the design and operation of arcade-style video games.

\section{Task Setup and Performance Data Collection}

We significantly modified the Asteroids game to make it suitable for experimental purposes (see Figure 1). It was important that the video-game hardware accept custom software and that it be possible to collect a complete data record of performance for later numerical analysis and visual "replay.",

To accomplish the first objective, we replaced the ROM containing the assembly-language game program with RAM. This RAM is made accessible to an Apple II + computer via a custom-built circuit board in the Apple, which is interfaced to a test connector on the Asteroids motherboard via a 50-conductor cable. The test connector allows access to all memory addresses in the video game and can temporarily electrically isolate the video game's 6502 processor. Thus the Apple II+ can write information directly to the video-game RAM. Task software is stored on floppy disks on the Apple II + and is downloaded as needed to the video game (via a small utility program). We used the Apple II + because it employs the same processor as does the video game (6502), which allows for straightforward assembly-language program development.

The second modification permits data collection and replay. In the video-game memory, there is an area devoted to keeping track of the positions and identities of all objects displayed on the screen and the status of each button (pressed or not pressed). This information is updated 60 times a second. A "frame" of data therefore represents a complete snapshot of the state of the task and a subject's actions for one 60th of a second. To collect these data frames on line, we added a second set of memory chips whose contents are updated simultaneously with the original area of memory. This second "shadow" RAM ( 1,024 bytes) can be accessed (read or written to) by the 80286 PC via a 34 -conductor cable connected to a 16-bit parallel circuit board in the PC.

Since the video-game processor and the $80286 \mathrm{PC}$ both have access to the same memory area, certain procedures are used to avoid contention in reading or writing to this area. The following procedure is used when a subject performs a task and the PC collects data: Every 60th of a second, access to the just-described memory area is blocked while the graphics processor updates the vector display and the game processor carries out some other calculations. At the beginning of this period, the task software signals the $\mathrm{PC}$ (using a hardware handshaking signal) to read the current frame of data. The PC then electrically isolates the RAM from the video game via a tri-state buffer that makes the memory available only to the PC. The PC then reads the relevant data $(30-130$ bytes, depending on the task), 16 bits at a time. When finished, the PC returns control of the memory to the video-game processor. This entire process occurs quickly enough so as not to interfere with the game processor's updating of the memory buffer. The PC stores the data on line in a RAM disk, since storing the data on a magnetic disk slows the process to a degree that interferes with the game processor's update cycle.

The collected data frame represents a complete description of the game state and the subject's game actions (buttonpresses) each 60th of a second. Such data records can be large; 2 min of performance occupy from 350,000 bytes to $1 \mathrm{MB}$, depending on the task. The data are archived eventually on an optical laser disk, which holds 240 MB per disk.

\section{Eye-Movement Data Collection}

In addition to collecting performance data, the system also collects synchronized eye-movement data. As can be seen in Figure 1, the subject performing a task sits facing the video-game cabinet with his or her head steadied by a chin- and forehead rest. The subject views a halfsilvered mirror tilted $60^{\circ}$ from horizontal. The center of the mirror is $38 \mathrm{~cm}$ from the subject's eyes. The game monitor is positioned under the mirror and angled $20^{\circ}$ from the horizontal. With this arrangement, the monitor appears to be directly in front of the subject's face at a distance of $66 \mathrm{~cm}$. At this distance, $1 \mathrm{~cm}$ on the game monitor equals $0.87^{\circ}$ of visual angle. Behind the mirror and invisible to the subject is a Panasonic CCTV infraredsensitive video camera (Model WV-CD20 with a 75-mm lens) and a near-infrared light, both aimed at the subject's left eye. The near-infrared light results from a collimated light source fitted with optical filters (Corning 7-69 and Kodak Wratten $87 \mathrm{c}$ ) to reduce heat and visibility.

The video output from the camera is fed into an ISCAN pupil/corneal reflection eye tracker (Model RK-426). ${ }^{2}$ This device processes the video signal to find the locations of the center of the pupil and the center of the corneal reflection of the infrared light. (The difference between these two positions is used to calculate where the subject is looking; see the Software section below.) These positions are located on a 256 (vertical) $\times 512$ (horizontal) pixel grid and are made available to a digital output port on the ISCAN tracker. The $80286 \mathrm{PC}$ reads these data, 60 times a second, via two 8-bit parallel boards. The eye-movement data are collected synchronously with the performance data (see the Software section).

\section{Performance and Eye-Movement Replay}

We designed the interface between the $\mathrm{PC}$ and the video game to allow collected data to be fed back to the video 
game to replay performance. This process reverses the method for collecting data: The PC now writes each frame of data to the shadow RAM, and the video-game software reads and displays the data. The user can specify the speed of replay, from fast motion to single frame. At 60 frames per second, the replay is identical to the original performance, since all the information originally present is displayed at the original presentation rate.

The system also permits one to replay a performance with a superimposed cross-hair representing the person's changing visual regard. After eye-movement data have been collected, they are processed by software that transforms the data into the same coordinate space as that of the objects in the video-game tasks. Once transformed, the eye-movement data are fed through the video game with the performance data, and a cross-hair is displayed in the appropriate $x, y$ position for each frame of data. This arrangement gives the researcher a unique opportunity to examine the coordination of eye movements with on-going action.

\section{SYSTEM SOFTWARE}

\section{Overview}

There were three important concerns that guided software development for this system: maintainability, flexibility, and manageability. Maintainability refers to the relative ease of modifying and extending the software across time and across programmers. Writing the software in structured, well-documented modules is critical in this regard. Most of the software for collecting and managing the data was written in Pascal; most programmers know this language, it encourages structured programming, and even novice programmers can make minor modifications.

Flexibility in the use of the software was another concern. It was important that the programs be usable for a variety of studies, ones not necessarily envisioned when the programs were developed. The goal was to "build the future' into the software's capabilities by minimizing the need for writing a new program for each new application. Although it is impossible to anticipate all possible future needs, we designed the software to maximize the user's ability to customize the operations of the programs. Thus, many of the programs behave differently, depending on how the user configures their operations.

A final concern was manageability, which refers to the problem of keeping track of the many output files created in this sort of system. In some studies, over 35 files are associated with a single testing session. To help manage this information, we developed strict conventions for how files are named. An eight-letter prefix uniquely identifies the study, the experimental condition, and the subject. The three-letter filename extension uniquely identifies the kind of information that is contained in the file. Thus programs that process data and create output data files "know" what kind of file extension should be used as input and automatically create the appropriate names for the output files. In addition, each original data file collected during per- formance has an associated "header" file (with the same prefix and an .HDR extension) that contains user-specified information on the file, subject, task, and session. Experimenters also keep written records of much of this information. We have found that redundancy of information is critical for recovering from user error and from lost information.

There are four categories of software: (1) task and session design; (2) calibration and data collection; (3) data editing, transformation, and replay; and (4) computing variables. Figure 3 presents a flowchart of the inputoutput relations among the various programs.
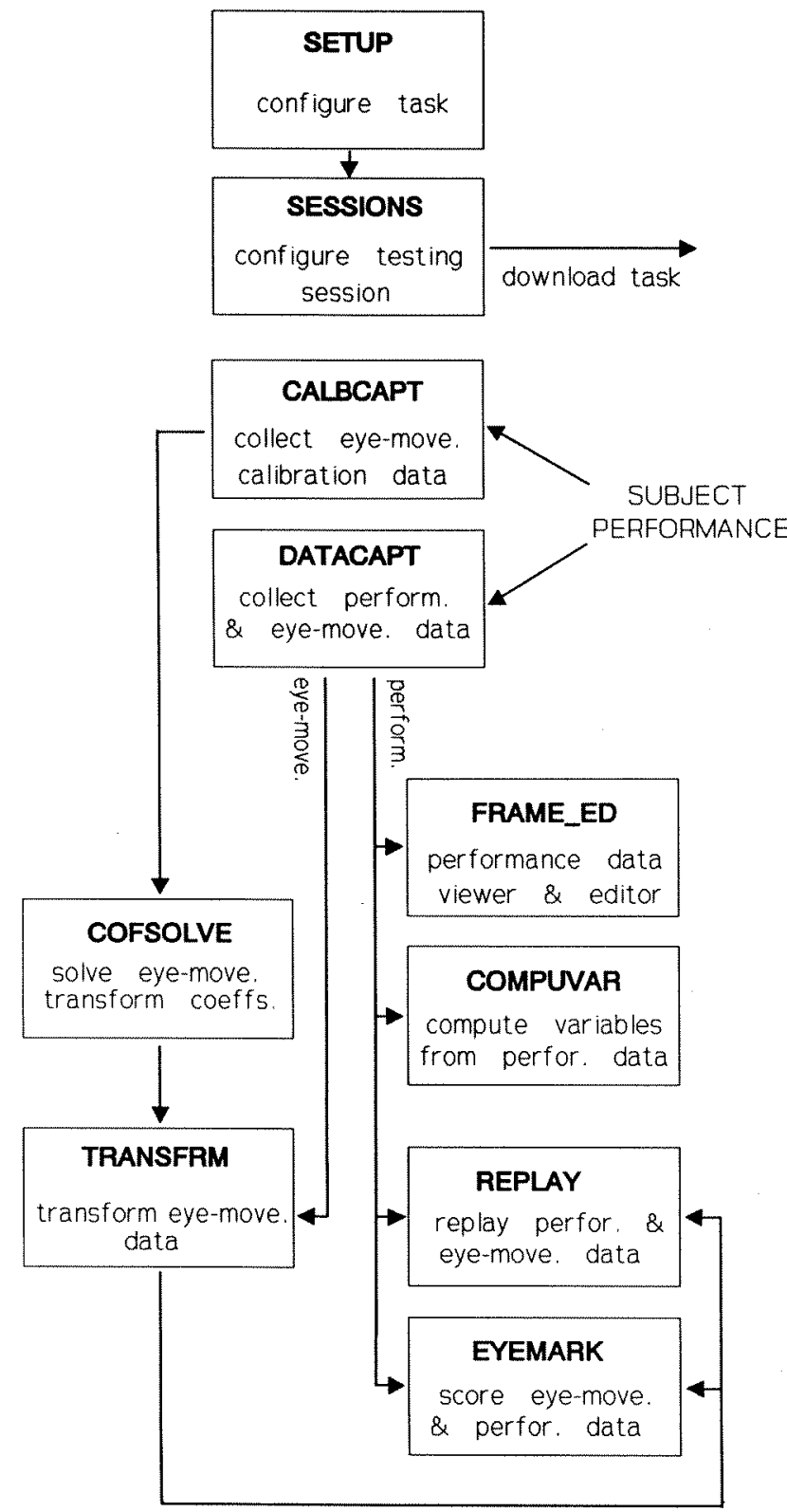

Figure 3. A flowchart of the input-output relations of the system software. See the text for a more detailed description of each program. 
Task and Session Design

Experimental tasks are configured on the Apple II + . For each type of task, a program called setup allows the user to set the values of several parameters that configure the task. Table 1 contains the modifiable parameters for the Fullgame task described above. These parameters are stored in a configuration file that is used to set the relevant values in the assembly-language task program that is downloaded to the video game. In addition, before the task is downloaded, the user can configure parameters of the design for a specific testing session (e.g., trial ordering and number of blocks of trials presented).

Four tasks developed thus far can be individually configured. The ability to customize the software allows these tasks to be used in a variety of ways without requiring a rewrite of the assembly-language code.

\section{Calibration and Data Collection}

Programs for eye-movement calibration and data collection run on the $80286 \mathrm{PC}$. The calibration program, calbcapt, instructs the video game to display a small dot at nine different locations on the video-game monitor (a $3 \times 3$ grid extending to the boundaries of the screen). When the subject looks at the current location of the dot, the experimenter signals the PC to collect 30 frames of visual fixation data $(x, y$ locations of the center of the pupil and the center of the corneal reflection of the infrared light) from the eye tracker. The PC immediately displays the mean and range of those data on the experimenter's monitor, so that the integrity of the data can be evaluated. If the data have too wide a spread, for example, there is too much noise in the system, and the experimenter might readjust the lighting or the threshold settings on the ISCAN eye tracker. Calibration data can then be recollected. After calibration, the data for each of the nine locations are stored in a file with a .CAL extension.

Before a subject begins a task, the experimenter runs a data-capture program (datacapt) on the 80286 PC that

Table 1

Modifiable Parameters for the Fullgame Task

Control Parameters
Ship rotation speed
Shot speed
Shot rate
Thrust force
Button function mapping
Environmental Parameters
Degree of thrusting "drag"
Number and sizes of asteroids at start of trial
Trajectories of asteroids (user-specifiable or random)
Speeds of asteroids
Autoreplacement of "hit" asteroids option
"Break-up" rules for hit asteroids
Presence or absence of "enemy saucers"
Experimental Parameters
Number of trials
Trial length
Type of between-trial feedback (score, no. of hits, no feedback)
Ordering of trials

first prompts the experimenter to enter information about the subject and the session, which is stored in the header file. Once the subject pushes the button to start the first trial, the data-capture program collects performance data from the video game in synchrony with visual data from the eye tracker at a rate of $60 \mathrm{~Hz}$. The timing of the data collection is driven by a 4.17 -msec nonmaskable interrupt used for timing the video refresh rate on the videogame monitor. During data collection, the program first reads a frame of data from the video game and then reads the data from the eye tracker. This scheme guarantees data synchronization within one frame. ${ }^{3}$ Data collection automatically halts during between-trial intervals, and the beginning and ending frame numbers for each trial are stored in the header file. Performance data are stored in a .DAT file, and the eye-movement data are stored in an .ISC file.

\section{Data Editing, Transformation, and Replay}

Data editing. Each frame of data from the video-game tasks contains the $x, y$ locations and identities of the objects displayed on the screen (e.g., the ship, asteroids, and shots) and the status of each of the buttons. This information is represented in hexadecimal format, since that is the numbering system used in the assembly-language task programs. A frame viewer/editor program (frame _ed) allows a user to display each frame in decimal format and permits the creation of new data files by "cutting and pasting'" together old ones.

Data transformation. Each frame of data collected from the eye tracker contains the $x, y$ coordinates of the positions of the centers of the pupil and the infrared corneal reflection. Subtracting one of these two positions from the other yields a single set of $x, y$ coordinates that are monotonically related to the person's true eye position. There are three problems with these data in terms of determining where the person is looking (foveating) on the task monitor (see Sheena \& Borah, 1981, for a more complete discussion of the issues involved in transforming eye-movement data). First, the "raw" data must be mapped onto the task monitor coordinate space. Second, a small amount of "crosstalk" from one axis can affect the measurements on the other axis. Finally, nonlinearities exist in the raw data, especially when the person is looking in corner locations. These nonlinearities are caused by the curved shape of the eyeball and individual variations in eyeball shape. Two programs transform the raw data to correct for these distortions.

The first program, cofsolve, uses the calibration data to determine the values of 18 coefficients (cf. Sheena \& Borah, 1981). The program first finds the mean $x, y$ "raw" eye-movement fixation points for each of the nine calibration points. The data from the five axial locations (i.e., excluding the four corner points) are then used to solve for the coefficients $a, b, c, d$, and $e$ in the following equation:

$$
A P_{\mathrm{h}}=a+b X+c X^{2}+d Y+e Y^{2},
$$

where $A P_{\mathrm{h}}$ is the actual horizontal position of the calibra- 
tion dot, $X$ is the raw horizontal eye position, and $Y$ is the raw vertical eye position. (This equation is for the horizontal component only; a comparable equation is used for the vertical component.) The first three terms of the equation map the raw positions to the known locations on the appropriate axis. The last two terms correct for crosstalk from the other axis. A total of 10 coefficients result from the horizontal and vertical components.

Cofsolve then determines the coefficients that correct for nonlinearities in the data by determining a separate "corner term" for each quadrant of the screen with the following equation:

$$
A P_{\mathrm{h}}=E P_{\mathrm{h}}+f_{n} X Y(n=1, \ldots 4),
$$

where $A P_{\mathrm{h}}$ is the actual horizontal position of the calibration dot (one of the four corners), $E P_{\mathrm{h}}$ is the estimated horizontal position resulting from Equation $1, X$ is the raw horizontal eye position, $Y$ is the raw vertical eye position, and $n$ is the quadrant. The equation is used for each quadrant, resulting in four coefficients for the horizontal component and four for the vertical component.

The PC stores the 18 coefficients in a .COF file that is used as input to transfrm, a program that takes the eyemovement data (.ISC) from a given task and transforms it using Equations 1 and 2. The resulting output data file (.EYE) is corrected for measurement error and coded in the video-game coordinate space. The effectiveness of the equations can be judged through examination of Figure 4. Displayed are raw fixation data and transformed data from a subject with a relatively high degree of distortion. The

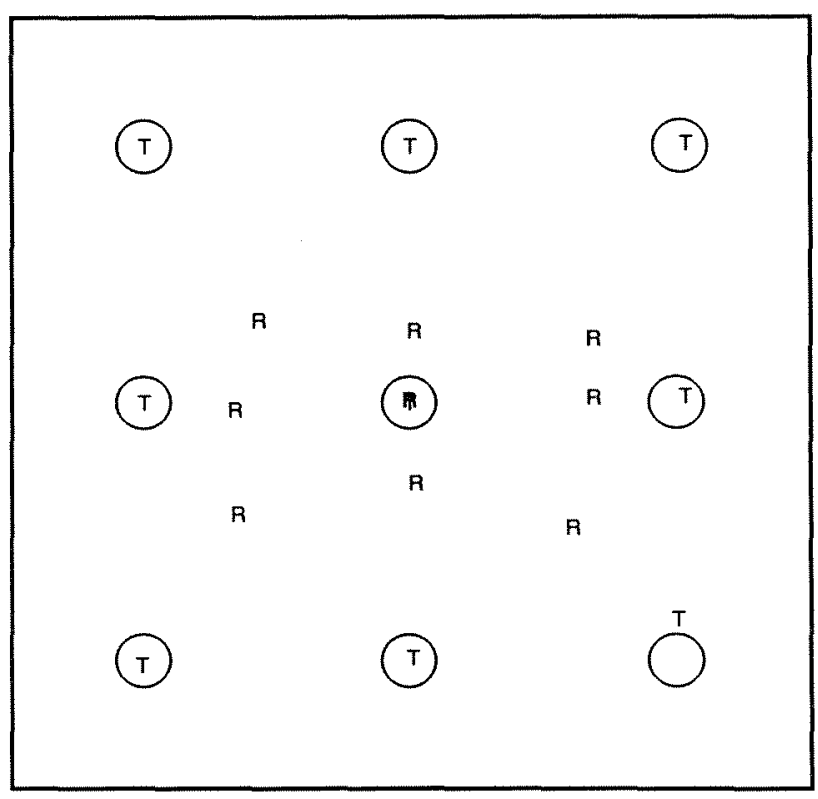

Figure 4. Eye-movement calibration data before and after the corrective transformation. The Rs show the raw eye-position data from the eye tracker. The circles show the actual locations of the calibration stimuli, mapped onto an arbitrary space. The Ts show the transformed estimates. Notice how most of the nonlinearities in the raw data are removed after the transformation.
Table 2

A Subset of the Performance Variables on the Fullgame Task Computed by the Compuvar Program

Buttonpressing frequencies (number of times pressed)

Buttonpressing durations (total number of frames each button pressed) Simultaneous buttonpressing (frequencies and durations of different button combinations)

Average durations between specific buttonpress sequences (e.g., between turning the ship and shooting)

Distance ship traversed across screen

Number of hits

Distances of hit targets from ship

Number of ship-target collisions

circles represent the locations of the calibration stimuli arranged in an arbitrary space around the center location. The Rs represent the raw data from the eye tracker, and the Ts show the transformed (corrected) looking positions. As shown, the transformation not only maps the raw fixation data onto the new space but also successfully corrects for most of the nonlinearities in the original data.

It is important to recognize that the .EYE file contains $x, y$ coordinates of visual regard for each data frame in the video game's coordinate space. Further coding is required to identify the timing of saccades, fixations, and smooth pursuits (as described in the next section).

Data replay. The collected data also can be replayed back through the video game by using the replay program. The researcher can replay each frame of data, one at a time, by entering the first frame number to be displayed and hitting the return key to advance to each successive frame. One also can replay entire trials at variable rates. Eye-movement data can be displayed along with the performance data and are represented by a small cross-hair. The cross-hair's location for each frame is determined by the value of the transformed eye-movement data.

\section{Computing Variables}

There are many variables that describe different aspects of a subject's performance for each of the video-game tasks. Specific sets of variables are used to describe differences in the organization of skill across expertise levels and to examine learning processes across time. Below, we describe two of the programs that compute these variables.

Compuvar is a program that computes up to 65 performance variables on the Fullgame task. The variables describe various aspects of performance for one trial. Table 2 presents some of the most common variables used. Each variable is calculated for each trial and output to an ASCII file that is used as input to standard statistics and graphing packages. The program computes the variables frame by frame until the end of a trial, at which point it adds one row of data to the output file. New variables can be added to the program relatively easily, through the addition of new procedures to the Pascal program.

The program can analyze a single file or an entire study by allowing the user to specify multiple files and addi- 
tional experimental variables associated with those files (e.g., age or sex of subject, session number, or experimental condition). Analyzing an entire study can involve processing several hundred files. For example, in one study (Roberts, Aman, \& Canfield, 1989) 24 subjects were tested 6 times each with 3 files collected on each visit (totaling 432 files). To help keep track of this potentially enormous database, compuvar makes an "audit trail" when it analyzes multiple files. The audit trail contains each file name, the file ordering in the analysis, and the user-specified variables associated with each file. The PC stores this information in a separate .TRK output file. This .TRK file can also be used later as input into another run of compuvar to specify the files to be processed for extracting additional variables.

Another analysis program, Eyemark, is used to code the eye-movement data and the correspondence between eye movements and task actions. The program aids a human coder in making decisions about the occurrence of saccades, fixations, smooth pursuit, and blinks. The coder examines two views of the data. Figure 5 shows one view of the eye-movement data as it is displayed on the 80286 PC. Frame number increases along the $x$-axis, and eye position is represented on the $y$-axis (using the transformed .EYE data). Two plots are displayed, one for the horizontal component of the eye position and another for the vertical component. A small circle on each plot high- lights the current frame. The user can advance the current frame forward or backward or can jump to a specific frame number. On the $x, y$ video-game monitor, the current frame of performance data is displayed with a crosshair showing the subject's point of visual regard.

The two views of the data maximize a user's ability to make accurate and reliable judgments when coding eye movements. On the PC monitor, the user views the eye's current position in relation to the preceding and following frames. A saccade is identified when there is a rapid change in position of the horizontal and/or vertical components; a fixation is identified when there is no consistent change on both components; and visual tracking occurs when one or both of the components change gradually (see Figure 5 for an example of each). On the $x, y$ monitor, the user views the eye's current position superimposed on the task screen. This view aids in the detection of saccades, fixations, and tracking in ambiguous cases. In addition, the user can make judgments concerning the location of the eye movements in relation to objects displayed in the task.

A coder marks the beginning of each type of eye movement on the PC by pressing a key (e.g., " $S$ " for saccade). This places a vertical color-coded line on the PC screen on the appropriate frame. The user can add up to two additional alphanumeric codes for each mark (e.g., indicating the location of a fixation on the task screen).

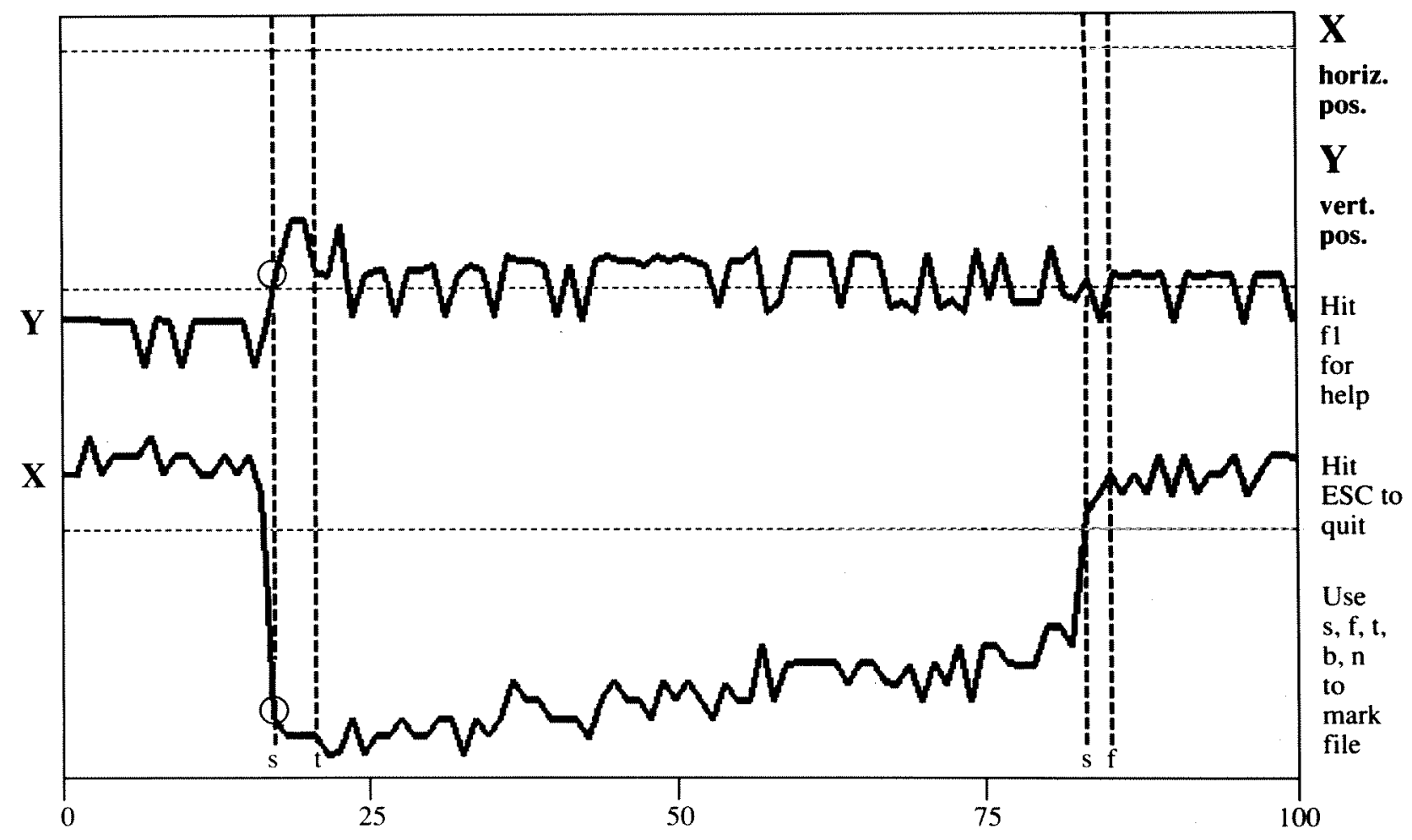

Frame: 17 x:72 y:523 marked as a Saccade

Figure 5. A representation of the screen displayed by the eyemark program on the $80286 \mathrm{PC}$. 
The PC stores these marks and associated frame numbers in a mark (.MRK) file. Also contained in the mark files are the frame numbers of the beginning and end of each buttonpress (left turn, right turn, thrust, and shoot). The mark file thus contains information on the timing and positioning of eye movements in relation to the timing and sequencing of game actions. Variables computed from this file concern the relation between eye movements and action. Examples include eye position during shooting, eye position during reorientation of the ship, and degree of visual anticipation of targets (by foveating on the target) before shooting.

\section{ILLUSTRATIVE DATA}

In the initial studies that we have done with the laboratory, we have focused on expert-novice differences in the real-time organization of perception-action skills and on the processes involved in the acquisition of expertise. The "grain level" of the data examined varies as a function of the issues under study. In some studies, large amounts of data are aggregated across time and across subjects, whereas in other studies, the moment-by-moment organization of performance is examined. We describe examples of both below.

Roberts et al. (1989) have examined developmental differences in how children acquire skill in the Fullgame task. We examined the performance of novices as they acquired skill in the absence of explicit instruction and explored several hypotheses concerning the reasons for developmental differences in learning. There were 24 subjects, equally divided across four age groups $(4,7,12$, and 20 years). The subjects came to the laboratory twice a week for 3 weeks. During each session, the subjects performed 1830 -sec trials of the task. The aggregated data for each age group are shown in Figure 6, averaged across the 18 trials per session. As can be seen (panel a), the 12 - and 20-year-olds averaged more successful hits per ship and showed more consistent improvement over sessions than did the younger age groups.

We hypothesized that one reason why younger children have difficulty in improving without instruction is that they do not spontaneously simplify the task in ways that maximize learning. In the present task, one critical simplification for learning to hit the moving targets is to refrain from moving the ship around the screen. The calculation of intercept times is complicated by a moving origin. As can be seen in panel $b$, the two younger age groups pushed the thrust button far more often than the older age groups did, and they actually increased thrusting time across sessions. These and other data suggest that the older age groups are more successful at reducing the task's complexities in ways that promote learning. More germane to the present purposes, these findings are examples of how aggregated data can be examined to explore differences between groups.

The laboratory is also used to study the organization of skill at a more microscopic level, especially in our
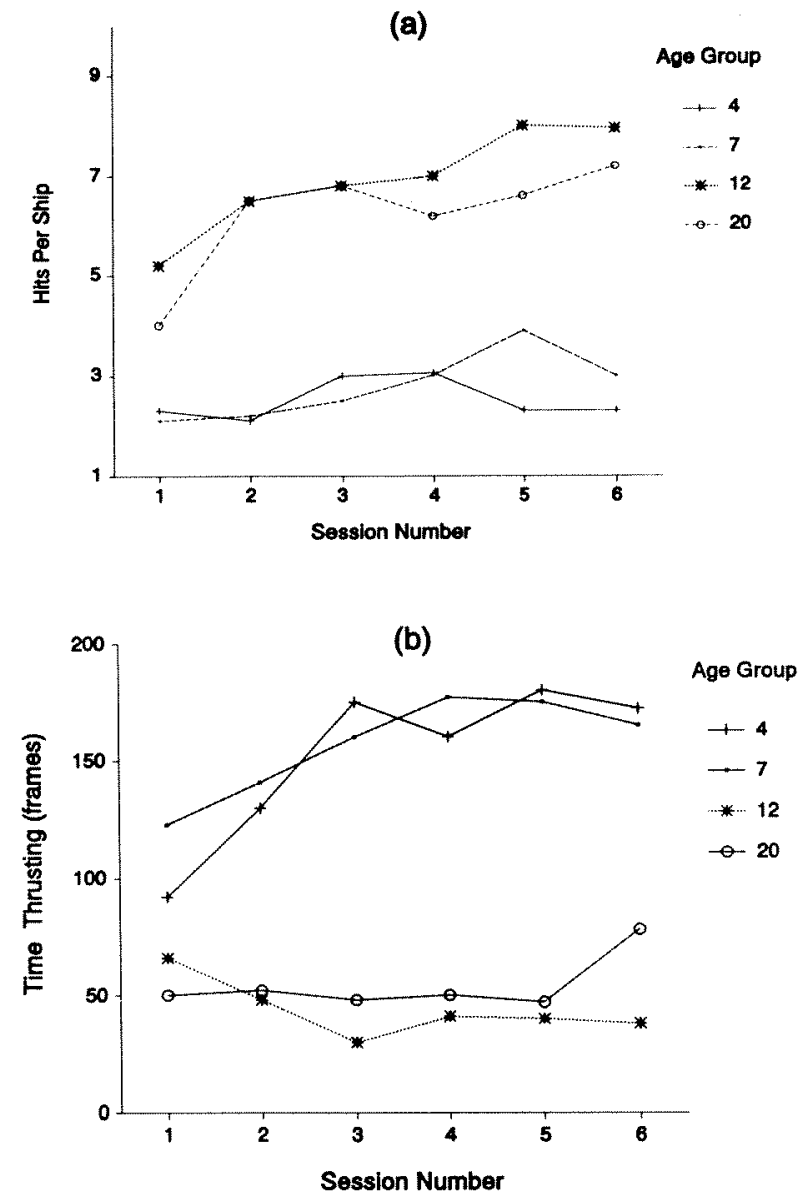

Figure 6. (a) Number of hits per ship as a function of age and session. For each session, the data are averaged across 1830 -sec trials. (b) Number of $1 / 30^{-}$-sec frames the thrust button was used as a function of age and session. For each session, the data are averaged across 1830 -sec trials.

studies of the relation between skilled action and perceptual processing. In one set of on-going studies, we are examining the coordination between visual information pickup, as reflected in eye movements, and the timing and sequencing of skilled action. Our goal is to find recurring patterns of coordination characteristic of different levels of expertise. Space limitations preclude a description of our work in the more complex tasks, so we present here characteristic data from the Interception Component task.

In this task, the subject (without moving or turning the ship) releases a single shot at a target moving across the screen. Since the targets vary in speed and distance from the ship, correct timing of the release of the shot cannot be judged reliably from one cue (e.g., target distance from the intercept point or elapsed time from the beginning of the trial). Instead, the subject must take account of the current target's distance and speed to make successful interceptions consistently. Eye movements may reflect how and when skilled performers extract this information. 


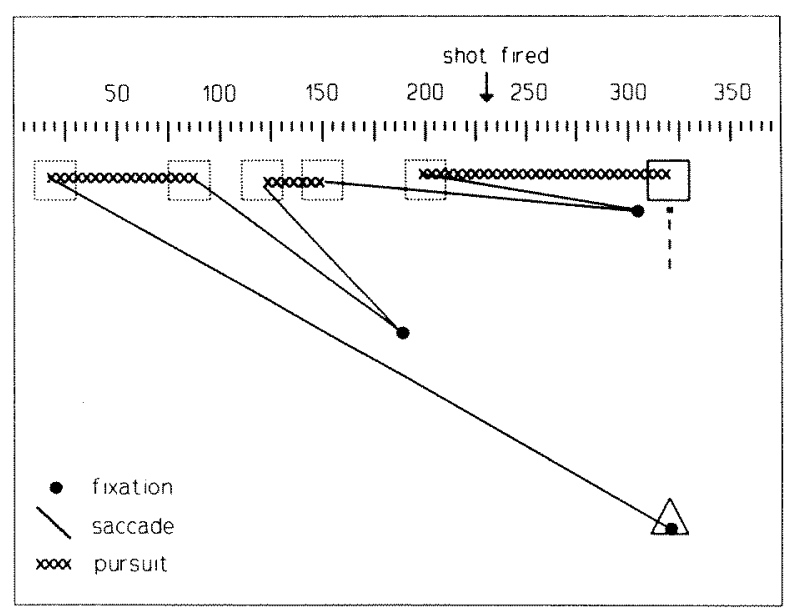

Figure 7. A representation of a subject's eye movements during a trial of the interception component task. The numbers across the top represent the target's position for each $1 / 60^{-\mathrm{Sec}}$ frame. The triangle at the bottom shows the position of the ship. The dotted boxes show the target's position at the beginning and end of each segment of time during which the subject visually tracks the target. The solid lines show saccades, the large dots show fixations, and the consecutive Xs show smooth pursuit. Also shown are the timing of the shot release and the positions of the target and shot immediately prior to interception. The figure shows the following sequence: The subject fixates the ship, then saccades to the target and tracks it, then saccades to a fixation location between the ship and the target, then saccades back to the target and tracks it, then saccades to a fixation position near the future intercept location, and finally returns to tracking the target, during which time the subject releases the shot.

Figure 7 shows the eye-movement patterns for a skilled player on a single trial of the interception task. The following sequence is illustrated: The subject first fixated on the ship and then made a saccade to the target and tracked it for 65 frames $(1,083 \mathrm{msec})$. He then made a saccade to fixate on a location between the target and the ship for 32 frames, after which he returned to track the target for another 30 frames. The subject then made a saccade to fixate on a location that was close to the future intercept position for 52 frames. Finally, he returned to track the target, during which time he released the shot. This scenario is typical of skilled performers: They tend to track the target most of the time and almost always when releasing the shot. Intermediate points of regard are either on the ship or midway between the ship and the target, depending on the target's distance from the ship and the time remaining before the shot is released. Finally, players often look at the intended intercept point toward the latter half of the trial, but rarely earlier. These and other initial findings suggest that eye-movement patterns reflect the on-line strategies employed in the timing and sequencing of skilled action. For the present discussion, these data show how our system can be used to study the real-time organization of perception and action.

\section{CONCLUSION}

We believe that our system provides a unique and powerful tool for studying the organization and acquisition of skill. The hardware and software are configured to permit flexibility and precision in task design and experimental setup as well as in data collection, representation, and analysis. Other researchers have shown the video game to be a useful context for studying skill (e.g., Bairstow, 1988; Cunningham, 1985; Donchin, 1989; Graham, Cook, Cohen, Phelps, \& Gerkovich, 1985; Richardson, Washburn, Hopkins, Savage-Rumbaugh, \& Rumbaugh, 1990), and our initial work supports this conclusion. Our system differs from other systems in several respects, but it is perhaps most unique in that it allows the examination of perceptual processing during skilled action. Perception and action are all too often isolated areas of inquiry, although researchers are increasingly recognizing their intricate interconnectedness in most everyday activities (cf. Schmidt, 1987; Turvey \& Carello, 1988). The present laboratory offers an approach to studying these relations that may be useful to other researchers. It is also our hope that some of the guidelines and techniques used in developing our system will be of value more generally to researchers in other disciplines who are contemplating developing real-time data collection/analysis systems.

\section{REFERENCES}

Arbib, M. A. (1980). Perceptual structures and distributed motor control. In V. B. Brooks (Ed.), Handbook of physiology: Sec. 6. The nervous system: Vol. 2. Motor control (pp. 1449-1480). Bethesda, MD: American Physiological Society.

Bairstow, P. J. (1988). Hand movement to moving targets: Planning and adjustments of speed and direction. In A. M. Colley \& J. R. Beech (Eds.), Cognition and action in skilled behavior (pp. 33-48). Amsterdam: North-Holland.

BENSON, J. B. (1990). The significance and development of crawling in human infancy. In J. E. Clark \& J. H. Humphrey (Eds.), Adwances in motor development research (Vol. 3, pp. 91-142). New York: AMS Press.

Book, W. F. (1908). The psychology of skill with special reference to its acquisition in typewriting. Missoula, MT: University of Montana.

Bryan, W. L., \& Harter, N. (1899). Studies on the telegraphic language. Psychological Review, 6, 345-375.

CARD, S. K., Moran, T. P., \& Newell, A. (1983). The psychology of human-computer interaction. Hillsdale, NJ: Erlbaum.

COOPER, W. E. (Ed.) (1983). Cognitive aspects of skilled typewriting. New York: Springer.

Cunningham, H. A. (1985). An Apple microcomputer-based laboratory system for the study of visual-motor behavior. Behavior Research Methods, Instruments, \& Computers, 17, 484-488.

Donchin, E. (1989). The learning strategies project: Introductory remarks. Acta Psychologica, 71, 1-16.

Graham, C., Cook, M. R., Cohen, H. D., Phelps, J. W. \& GerKoVICH, M. M. (1985). STAR: A unique embedded performance assessment technique. Behavior Research Methods, Instruments, \& Computers, 17, 642-651.

Hofsten, C. von (1985). Perception and action. In M. Frese \& J. Sabini (Eds.), Goal directed behavior: The concept of action in psychology (pp. 80-96). Hillsdale, NJ: Erlbaum. 
Hofsten, C. von, \&ONNQVist, L. (1988). Preparation for grasping an object: A developmental study. Journal of Experimental Psychology: Human Perception \& Performance, 14, 610-621.

LASHLEY, K. S. (1951). The problem of serial order in behavior. In L. Jeffress (Ed.), Cerebral mechanisms in behavior: The Hixon symposium (pp. 112-135). New York: Wiley.

Richardson, W. K., Washburn, D. A., Hopxins, W. D., SavageRumbaugh, E. S., \& Rumbaugh, D. M. (1990). The NASA/LRC computerized test system. Behavior Research Methods, Instruments, \& Computers, 22, 127-131

Roberts, R. J., JR., Aman, C., \& CANFIEld, R. (1989, April). Developmental differences in learning a new skill: The role of self-imposed constraints. Paper presented at the biennial meeting of the Society of Research in Child Development, Kansas City, MO.

SCHAFFER, L. H. (1981). Performances of Chopin, Bach and Bartok: Studies in motor programming. Cognitive Psychology, 13, 326-376.

ScHмIDT, R. A. (1987). The acquisition of skill: Some modifications to the perception-action relationship through practice. In H. Heur \& A. Sanders (Eds.), Perspectives on perception and action (pp. 77104). Hillsdale, NJ: Erlbaum.

Sheena, D., \& BORAH, J. (1981). Compensation for some second order effects to improve eye position measurements. In D. F. Fisher, R. A. Monty, \& J. W. Senders (Eds.), Eye movements: Cognition and visual perception (pp. 257-268). Hillsdale, NJ: Erlbaum.
Turvey, M. T., Carello, C. (1988). Exploring a law-based ecological approach to skilled action. In A. M. Colley \& J. R. Beech (Eds.), Cognition and action in skilled behavior (pp. 191-203). Amsterdam: North-Holland.

WiNTER, D. A. (1979). Biomechanics of human movement. New York: Wiley.

\section{NOTES}

1. The software described in this article is available from the first author. Send a blank disk and prepaid mailer. Some of the programs were designed specifically for the hardware described in the paper, although many could be easily modified to work with other systems.

2. Information on the ISCAN corneal reflection eye tracker can be obtained by contacting Iscan Inc., 755A Concord Avenue, P.O. Box 2076, Cambridge, MA 02238 .

3 . The eye-movement data actually lag 4 frames behind the videogame data. A two-frame delay is caused by processing time in the camera circuitry, and an additional two-frame delay is caused by processing in the ISCAN tracker. The combined delay is constant and corrected for after data collection.

(Manuscript received September 25, 1990; revision accepted for publication March 25, 1991.)

\section{Articles in press Behavior Research Methods, Instruments, \& Computers}

The following is a list of forthcoming Behavior Research Methods, Instruments, \& Computers articles that are currently in press. They are given in approximate order of acceptance. Each entry includes the name and address of the author with whom to communicate for further prepublication information.

"A computerized system for analyzing linear locomotion" by E. Scherer, R.E. McNicol, \& M.J. Capel (E.S., Dept. of Fisheries and Oceans, Freshwater Institute, 501 University Crescent, Winnipeg, MB, Canada R3T 2N6)

"Response key input via the IBM PC/XT/AT's parallel printer port" by E.C. Dalrymple-Alford (Dept. of Psychology, Univ. of Guelph, Guelph, ON, Canada N1G 2W1)

"Color-vision demonstrations on an IBM PC/AT with VGA" by $H$. Irtel (Universität Regensburg, Institut für Psychologie, D-8400 Regensburg, Germany)

"Shuffling arrays: Appearances may be deceiving" by N.J. Castellan, Jr. (N.J.C., Dept. of Psychology, Indiana Univ., Bloomington, IN 47405)

"Visual word-recognition thresholds for screen-fragmented names of the Snodgrass and Vanderwart pictures" by J.G. Snodgrass \& M. Poster (J.G.S., Dept. of Psychology, New York Univ., 6 Washington Pl., Rm. 857, New York, NY 10003)

"CATECHOLLISION: A game to teach catecholamine synthesis for IBM-PCs and compatibles"' by W.J. Wilson \& J.A. Cook (W.J.W., Dept. of Psychological Sciences, Indiana Univ.-Purdue Univ., 2101 Coliseum Blvd. East, Fort Wayne, IN 46805)

"A LOTUS 1-2-3-based system for recording and maintaining body weight of laboratory animals" by J.S. Ali, V.B. Olszyk, D.D. Dunn, K.A. Lee, S.M. Kendall, R.R. Rhoderick, \& P.J. Bushnell (J.S.A., Neurotoxicology Division, MD 74B, US Environmental Protection Agency, Research Triangle Park, NC 27711)

"Lateralization in haptic processing: An apparatus to analyze manual strategies" by J. Fagot, B. Arnaud, M. Chiambretto, \& R. Fayolle (J.F., CNRS, Lab. de Neurosciences Fonctionnelles, 31 chemin Joseph Aiguier, 13409, Marseille Cedex 09, France)

"Analysis of the circadian rhythm of body temperature"' by R. Refinetti (Dept. of Biology, Univ. of Virginia, Charlottesville, VA 22901)

"Sample properties of principal components using gas"' by A. Narayanan (Dept. of Decision \& Information Systems, Graduate School of Business, Indiana Univ., Bloomington, IN 47405)

"An EXCEL macro for transformed and weighted averaging" by S.A. Klein (School of Optometry, Univ. of California, Berkeley, CA 94720)

"Picture Perception Lab: A program for picture perception experiments on the Macintosh II"' by S.W. Kohlmeyer (Dept. of Psychology, Elliott Hall, Univ. of Minnesota, Minneapolis, MN 55455)

"Tachistoscopic software for Macintosh computers" by T.A. Busey (Dept. of Psychology, Univ. of Washington, Seattle, WA 98195)

"Tachistoscopic software for the Macintosh" by C. Barron (Dept. de psychologie, Univ. du Quebec à Montreal, P.O. 8888 Succ 'A' Montreal, Quebec, Canada H3C 3P8)

"A versatile, user-friendly tachistoscope for the Macintosh" by J.M. Doenias, S.E. Langland, \& D. Reisberg (D.R., Dept. of Psychology, Reed College, Portland, OR 97202)

"An automated exercise wheel for primates" by C.J. Sherry \& S.H. Constable (S.H.C., Armstrong Laboratory, AL/CFTO, Brooks Air Force Base, TX 78235) 